\title{
Cell Separation with Hybrid Magnetic Levitation- Based Lensless Holographic Microscopy Platform
}

\author{
Kerem Delikoyun, Sena Yaman, Muge Anil-Inevi, Engin Ozcivici, H. Cumhur Tekin \\ Department of Bioengineering \\ Izmir Institute of Technology \\ Izmir, Turkey \\ cumhurtekin@iyte.edu.tr
}

\begin{abstract}
Separation of target cells in a heterogeneous solution is of great importance for clinical studies especially for immunology and oncology. Separated cells can be used for diagnostic applications ranging from whole blood counting to isolation of circulating tumor cells (CTC) for personalized medicine. Recent separation technologies rely on labelling and identifying target cells with variety of labelling principle such as fluorescence or magnetic tags. However, they require laborintensive processes, long analysis time, and expensive chemical reagents and instrumentation. Hence, their usage is limited to wellequipped centralized laboratories. There is a need for a rapid, sensitive, low-cost and automated cell separation technology to disseminate usage of this technology even in rural areas. Magnetic levitation is a powerful cell separation method, which distinguishes cells based on their levitation heights depending on cell density. However, magnetic levitation-based separation technologies require traditional, bulky and expensive microscopes for analysis. Lensless digital inline holographic microscopy (LDIHM) systems are composed of a simple illumination system containing an LED, a pinhole, and an imaging sensor for high-resolution microscopic imaging, which eliminates needs of highly fragile and expensive optics as in traditional microscopy. Here, we introduced a novel hybrid and portable cell separation platform, where magnetic levitation technology is integrated with LDIHM system for automated analysis of cell levitation heights. Using this platform, three different cell lines are successfully separated. Live and dead cells having distinguished levitation heights can be also identified in the platform.
\end{abstract}

Keywords - Cell separation, magnetic levitation, density measurement, lensless digital inline holographic microscopy, portable analysis

\section{INTRODUCTION}

Cell separation plays a huge role in both clinical and research-based laboratories for different applications including whole blood counting and isolation of circulating tumor cells (CTCs) [1]. Various cell separation technologies are available, and they are divided into two as label-dependent and label-free technics [2]. For instance, fluorescent or magnetic labels are used to tag cells in label-dependent cell separation techniques. However, they require costly and time-consuming protocols, and expensive equipment. Heterogeneous cell surface markers can also make separation process challenging for labeldependent techniques. On the other hand, label-free techniques offer a great potential for cell separation applications, which physical cell parameters such as size, density, charge, elasticity, etc. can be used for separation [3]. These techniques can offer low-cost, rapid and portable analysis, which can disseminate usage of cell separation technologies. One of the most exciting label-free technique that offers a simple and cost-effective way of separating cells is magnetic levitation [4]. In the magnetic levitation system, cells are separated under an inhomogeneous magnetic field created by two opposing magnets based on their density in a short time and at single-cell level [5]. Different cell lines show inherent differences in density that can be easily separated by using magnetic levitation principle. However, benchtop microscopes are required for magnetic levitation technique for assessment of cell levitation height $[4,6,7]$. Traditional microscopes are bulky, composed of expensive and precise optics such as lenses, mirrors and filters to achieve highresolution images which make the image quality dramatically dependent upon configuration of instrument and skills of operating personnel. On the other hand, lensless digital inline holographic microscopy (LDIHM) eliminates costly and fragile optics by replacing simple illumination and recording scheme, which provides low-cost, high-resolution and portable microscopic imaging [8, 9]. Furthermore, recorded images in LDIHM can be analyzed using fully automated digital image processing algorithms, which eliminates also human errors.

In this study, we present a new hybrid and portable cell separation platform to separate and identify cells based on their densities using magnetic levitation principle and to analyze automatically cell levitation heights using LDIHM.

\section{EXPERIMENTAL METHODS}

\section{A. Platform Design}

Our hybrid platform is the combination of two separate technologies relying on firstly separating cells in the capillary channel based on magnetic levitation principle and consecutively monitoring them to evaluate their levitation heights using LDIHM. The components of the platform are assembled with a 3D-printed body (Fig. 1a) [10].

Magnetic levitation system is composed of two opposing magnets (N52 grade neodymium magnets, $50 \mathrm{~mm}$ length $\times 2$ $\mathrm{mm}$ width $\times 5 \mathrm{~mm}$ height $)$ and a microcapillary (50 $\mathrm{mm}$ length $\times 1 \mathrm{~mm}$ width $\times 1 \mathrm{~mm}$ height) where cells spiked in paramagnetic medium are introduced.

In LDIHM, illumination is achieved using $1 \mathrm{~W}$ LED with a central wavelength of $650 \mathrm{~nm}$. The light is spatially filtered by a relatively large pinhole with a $100-\mu \mathrm{m}$ diameter to get quasimonochromatic light. Pinhole is located about $5 \mathrm{~cm}$ away from 
the surface of the glass microcapillary. Light diffracted on cells (object wave) is recorded along with constant phase background illumination (reference wave) as holograms on the imaging sensor placed in a close proximity $(<1 \mathrm{~mm})$ to glass microcapillary. Sony IMX219 complementary metal oxide semiconductor (CMOS) imaging sensor array is connected to Raspberry Pi 3 Model $\mathrm{B}+$ to capture image with custom developed Python script. Active area of sensor array is $3280(\mathrm{H}) \times 2464(\mathrm{~W})$ pixels with a pixel size of $1.12 \mu \mathrm{m}$.

\section{B. Magnetic Levitation Principle}

Cells come to an equilibrium levitation height where magnetic and buoyancy forces are balanced (Fig. 1b). The final levitation height of a cell can be determined by [4]:

$$
\frac{V \cdot\left(\chi_{c}-\chi_{m}\right)}{\mu_{0}}(\vec{B} \cdot \nabla) \vec{B}+V\left(\rho_{c}-\rho_{M}\right) g=0
$$

with the volume of the cells $(V)$, the magnetic susceptibility of cells $\left(\chi_{c}\right)$ and paramagnetic medium $\left(\chi_{m}\right)$, the magnetic flux density $(\vec{B})$, the volumetric density of cells $\left(\rho_{c}\right)$ and the medium $\left(\rho_{M}\right)$, the gravitational acceleration $(g)$, and the permeability of the medium $\left(\mu_{0}\right)\left(1.2566 \times 10^{-6} \mathrm{~kg} \cdot \mathrm{m} \cdot \mathrm{A}^{-2} \cdot \mathrm{s}^{-2}\right)$.

a

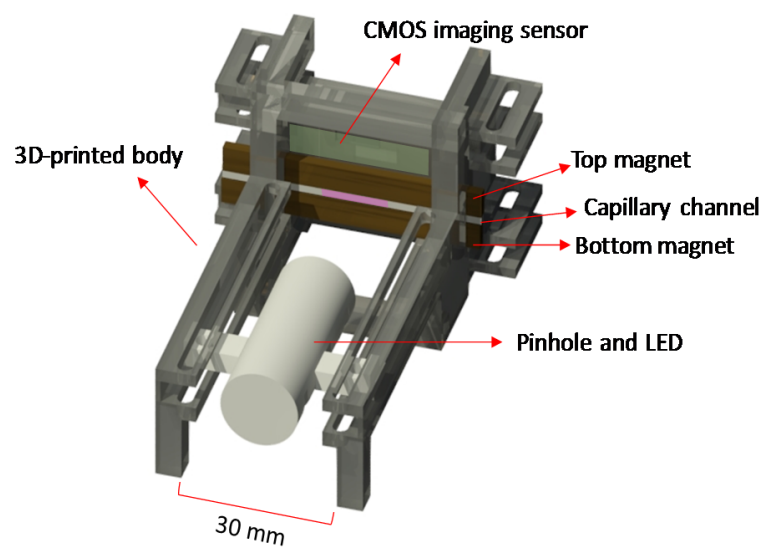

b

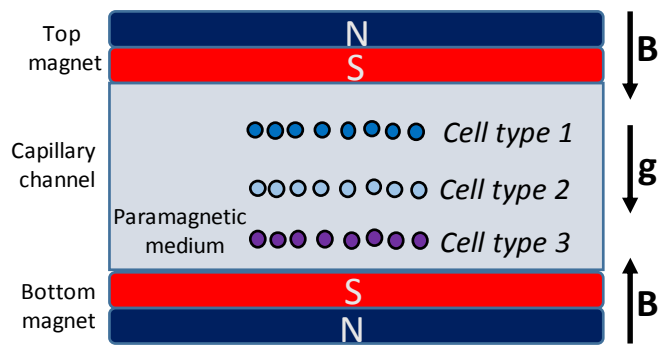

Fig. 1. (a) Illustration of the hybrid platform. (b) The schematic representation of magnetic levitation-based cell separation. Cells with higher density levitate at lower heights. $\mathbf{B}$ and $\mathbf{g}$ denote magnetic induction and gravitational acceleration, respectively.
In this equation, $\chi_{c}$ can be neglected because cells have lower magnetic susceptibility than the surrounding medium. Hence, differences in cell levitation heights depend on the density differences, where cells are positioned in the capillary channel from bottom to top as high to low density cells (Fig. 1b).

\section{Sample Preparation}

D1 bone marrow stem cell [11], U937 monocyte and MDAMB-231 breast cancer cell lines were examined in the platform. Live and dead D1 bone marrow stem cells were also investigated. Dead cells are obtained by introducing $50 \%(\mathrm{v} / \mathrm{v})$ dimethyl-sulfoxide to cell environment. For all experiments, cell samples were prepared with $1 \times 10^{5}$ cells $/ \mathrm{mL}$ in phosphate buffered saline (PBS) containing 30 or $100 \mathrm{mM}$ Gadavist (Gd) and $1 \%(\mathrm{w} / \mathrm{v})$ Pluronic F-127. After that, $40 \mu \mathrm{L}$ of cell sample was loaded into the glass microcapillary in the platform.

\section{LDIHM Imaging Technique}

LDIHM is an imaging technique, which records superposition of light diffracted from the object and background illumination with constant phase. Hence, the wave front coming from the light source to the imaging sensor is the interference pattern of waves containing information regarding the light source and the object placed between the light source

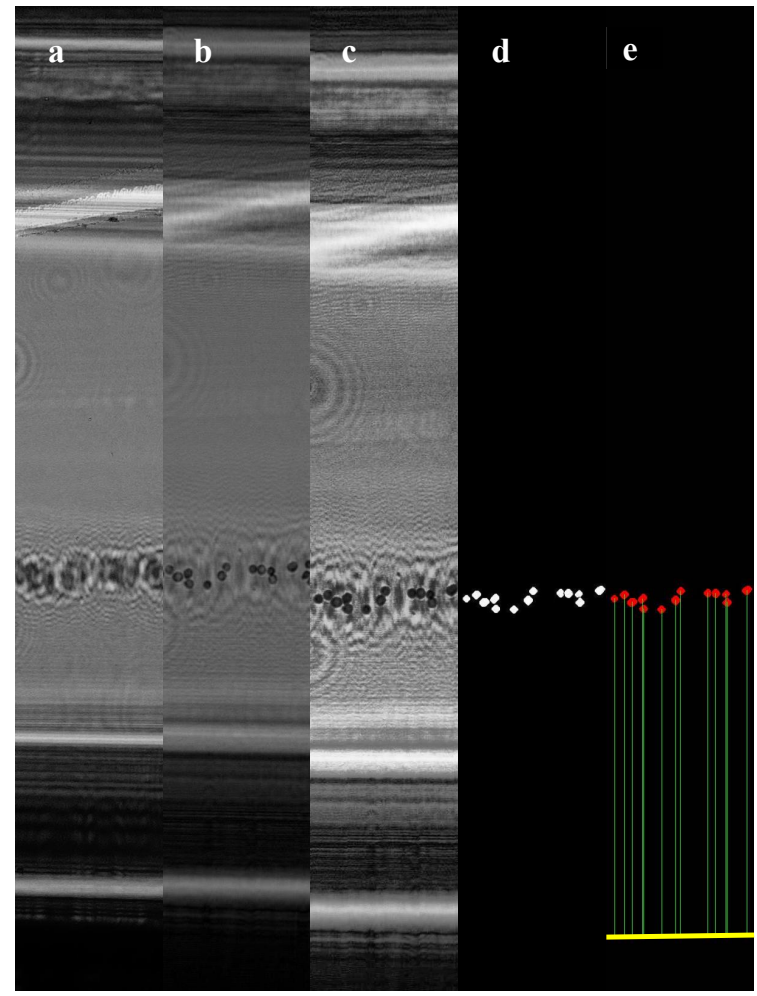

Fig. 2. Illustration of digital processing steps to determine levitation heights of MDA-MB-231 cells spiked in $30 \mathrm{mM}$ Gd. (a) Capturing of cell holograms, (b) amplitude of reconstructed image, (c) enhancement and tilting of the image, (d) creating binary mask, (e) finding cells (red) and their levitation heights (green lines) with respect to bottom magnet (yellow line). 
and the imaging sensor. Recorded holograms on the imaging sensor (Fig. 2a) were subjected to a reconstruction process, which uses back-propagation with a spatial transfer function for obtaining real object images [8] as follows:

$$
\Psi_{P}(x, y ; z)=\mathcal{F}^{-1}\left\{\mathcal{F}\left\{\Psi_{P o}(x, y)\right\} H\left(k_{x}, k_{y} ; z\right)\right\}
$$

In this equation, Fourier transformation of recorded image denoted as $\mathcal{F}\left\{\Psi_{P o}(x, y)\right\}$ is multiplied by spatial transfer function, $H\left(k_{x}, k_{y} ; z\right)$ along $z$-axis and then, inverse Fourier transformation is taken to reconstruct image at the object plane by back-propagation, where the object is placed at a specific distance far away from the imaging sensor along the $\mathrm{z}$-axis. The reconstructed image is complex-valued, and amplitude (Fig. 2b) and phase information of each pixel can be calculated from this image. The transfer function can also be calculated as follows:

$$
H\left(k_{x}, k_{y} ; z\right)=\left[-j k_{o} z \sqrt{1-\frac{\left(p \Delta_{k x}\right)^{2}}{k_{o}^{2}}-\frac{\left(q \Delta_{k y}\right)^{2}}{k_{o}^{2}}}\right]
$$

where $k_{x}$ and $k_{y}$ are indices in Fourier domain corresponded in $\mathrm{x}$ and $\mathrm{y}$ in spatial domain, $k_{o}$ is the wavenumber, and $\Delta_{k x}$ and $\Delta_{k y}$ are sampling periods depending on pixel sizes of the image sensor in $\mathrm{x}$ and $\mathrm{y}$ direction, respectively.

\section{E. Automatically Determining Levitation Heights of Cells}

First, reconstructed image was processed for determining spatial and angular position of bottom magnet using Hough transformation. Then, the image was tilted respectively to align bottom magnet with respect to the horizontal axis. Afterwards, image contrast was enhanced (Fig. 2c), and binary mask was used to find cells in the image. Each white region corresponding to cells were detected on the generated binary image using Sauvola thresholding (Fig. 2d). Finally, levitation heights of cells were calculated with respect the bottom magnet position (Fig. 2e) [12]. In-house-developed MATLAB program was used for all digital image-processing steps.

\section{F. Statistical Analysis}

For each levitation experiments, levitation heights of $40 \mu \mathrm{L}$ of $10^{5}$ cells $/ \mathrm{mL}$ solution were examined. Data pairs were analyzed using an ordinary one-way ANOVA with post-hoc correction for three cell lines. For viability analysis, levitation heights of viable and dead D1 cells were compared with an unpaired t-test. Statistical difference was defined as $\mathrm{P}<0.05$.

\section{RESULT AND DISCUSSION}

D1 bone marrow stem cell, U937 monocyte and MDA breast cancer cells spiked in $30 \mathrm{mM}$ Gd solution were analyzed in the platform. After 10 minutes, the average $( \pm$ SD) levitation heights of D1, MDA-MB-231 and U937 cells were at $548 \pm 31 \mu \mathrm{m}, 535 \pm 23 \mu \mathrm{m}, 524 \pm 30 \mu \mathrm{m}$ respectively, with significant differences (all $\mathrm{P}<0.05$ ) between groups (Fig. 3). Hence, each cell lines show distinct levitation profile and can be separated from each other in this platform.

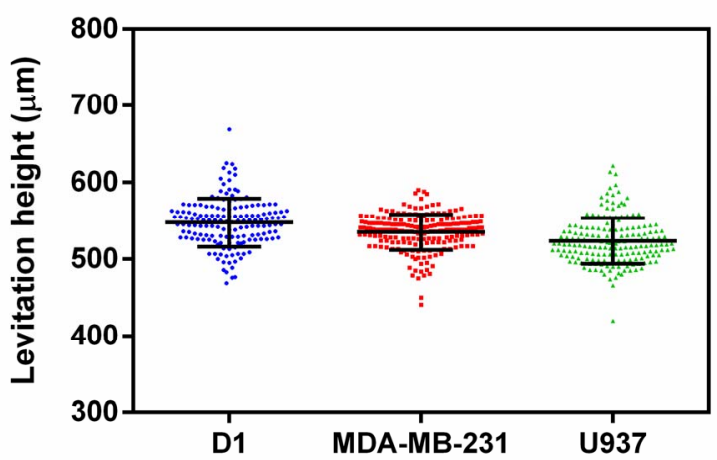

Fig. 3. Levitation height profiles of D1 bone marrow stem, MDA breast cancer and U937 monocyte cells spiked in $30 \mathrm{mM}$ Gd solution.

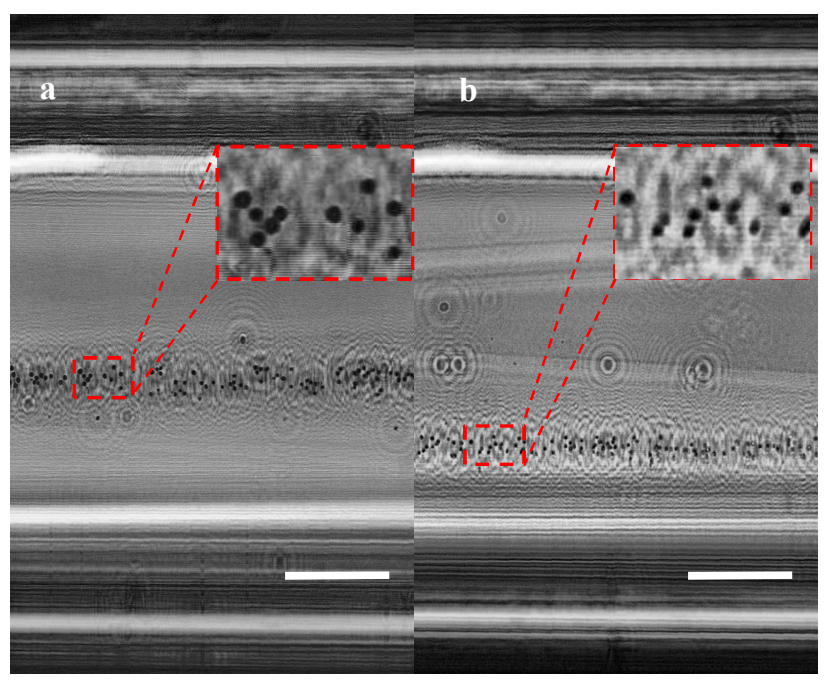

Fig. 4. Levitation height profiles of (a) live and (b) dead D1 bone marrow stem cells spiked in $100 \mathrm{mM}$ Gd solution. Scale bars are $300 \mu \mathrm{m}$.

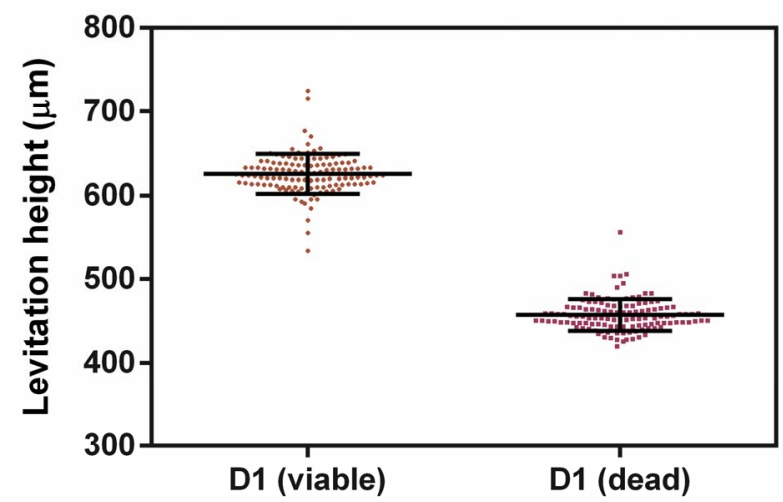

Fig. 5. Levitation height profiles of live and dead D1 bone marrow stem cells sampled in Fig. 4. 
When dead D1 bone marrow stem cells were levitated in 30 $\mathrm{mM}$ Gd solution, they were accumulated at the bottom of the microcapillary. Therefore, we increased Gd concentration to $100 \mathrm{mM}$ in order to collect dead cells at higher levitation heights (Fig. 4). After suspending dead and live D1 bone marrow stem cells in $100 \mathrm{mM}$ Gd-based levitation medium, significant difference in cell levitation heights between this group of cells was determined after 10 min of levitation. According to the results (Fig. 5), dead cells were collected at lower levitation heights $(457 \pm 19 \mu \mathrm{m})$ compared to viable cells $(626 \pm 23 \mu \mathrm{m})$. Hence, dead cells were measured as denser than the live cells in the platform. This could be due to the physiological changes during cell death [13].

The experiments revealed that our platform could differentiate different cell lines easily. The platform can also be used to distinguish dead cells from the live ones. The platform can monitor and measure levitation heights of single cells automatically. The levitation height of cells and so the density of cells can be used as a biomarker in the platform to distinguish and separate single cells rapidly from heterogeneous cell populations without using any labels.

\section{CONCLUSION}

In this study, we introduced a novel hybrid platform for separation of cells based on their density differences. In the platform, cells were magnetically levitated, and they were imaged using LDIHM to determine their levitation heights depending on cell densities. This hybrid platform enables rapid, sensitive and cost-effective cell identification and separation with low volume sample. Furthermore, the platform has robust, low-cost $(<100 \$)$ and compact design $(6 \mathrm{~cm} \times 6 \mathrm{~cm} \times 10 \mathrm{~cm})$, which also provides low maintenance cost. In the platform, all imaging and analysis processes are automated and standardized meaning that device does not have to be operated by skilled technical personnel. Thus, this hybrid platform could increase the use of cell separation technologies by non-expert individuals and patients, and it could be very convenient for variety of applications for point-of-care testing.

\section{ACKNOWLEDGEMENT}

Authors would like to thank The Scientific and Technological Research Council of Turkey (116M298) for funding this work. K.D. acknowledges the support of Turkish Council of Higher Education for 100/2000 CoHE doctoral scholarship.

\section{REFERENCES}

[1] A. Dalili, E. Samiei, and M. Hoorfar, "A review of sorting, separation and isolation of cells and microbeads for biomedical applications: microfluidic approaches," Analyst, vol. 144, pp. 87-113, Dec 172018.

[2] C. W. Shields, C. D. Reyes, and G. P. Lopez, "Microfluidic cell sorting: a review of the advances in the separation of cells from debulking to rare cell isolation," Lab Chip, vol. 15, pp. 1230-49, Mar 72015.

[3] D. R. Gossett, W. M. Weaver, A. J. Mach, S. C. Hur, H. T. Tse, W. Lee, et al., Label-free cell separation and sorting in microfluidic systems," Anal Bioanal Chem, vol. 397, pp. 3249-67, Aug 2010.

[4] S. Yaman, M. Anil-Inevi, E. Ozcivici, and H. C. Tekin, "Magnetic ForceBased Microfluidic Techniques for Cellular and Tissue Bioengineering," Front Bioeng Biotechnol, vol. 6, p. 192, 2018.

[5] O. Sarigil, M. Anil-Inevi, E. Yilmaz, G. Mese, H. C. Tekin, E. Ozcivici, "Label-free density-based detection of adipocytes of bone marrow origin using magnetic levitation," Analyst, vol 144(9), pp. 2942-2953, March 11, 2019 .

[6] M. Anil-Inevi, E. Yilmaz, O. Sarigil, H. C. Tekin, E. Ozcivici, "Single cell densitometry and weightlessness culture of mesenchymal stem cells using magnetic levitation," In:Methods in Molecular Biology. Humana Press, pp 1-11, April 25, 2019

[7] M. Anil-Inevi, S. Yaman, A. A. Yildiz, G. Mese, O. Yalcin-Ozuysal, H. C. Tekin, et al., "Biofabrication of in situ Self Assembled 3D Cell Cultures in a Weightlessness Environment Generated using Magnetic Levitation," Sci Rep, vol. 8, p. 7239, May 82018.

[8] K. Delikoyun, E. Cine, M. Anil-Inevi, M. Ozuysal, E. Ozcivici, H. C Tekin, "Lensless Digital In-line Holographic Microscopy for Space Biotechnology Applications," 9th International Conference on Recent Advances in Space Technologies, 11-14 June 2019.

[9] A. C. Sobieranski, F. Inci, H. C. Tekin, M. Yuksekkaya, E. Comunello, D. Cobra, et al., "Portable lensless wide-field microscopy imaging platform based on digital inline holography and multi-frame pixel superresolution," Light: Science \&Amp; Applications, vol. 4, p. e346, 10/23/online 2015.

[10] S. Yaman, K. Delikoyun, H. C. Tekin, "Magnetic Levitation-Based Protein Detection Using Lensless Digital Inline Holographic Microscopy," The 23rd International Conference on Miniaturized Systems for Chemisty and Life Sciences, 27-31 October 2019.

[11] O. Baskan, G. Mese, and E. Ozcivici, "Low-intensity vibrations normalize adipogenesis-induced morphological and molecular changes of adult mesenchymal stem cells," Proceedings of the Institution of Mechanical Engineers, Part H: Journal of Engineering in Medicine, vol. 231, pp. 160$168,2017$.

[12] H. C. Tekin, "Determining densities of microparticles in a magnetic levitation platform using automated digital image analysis," in 2018 26th Signal Processing and Communications Applications Conference (SIU), pp. 1-4, 2-5 May 2018.

[13] N. G. Durmus, H. C. Tekin, S. Guven, K. Sridhar, A. Arslan Yildiz, G. Calibasi, et al., "Magnetic levitation of single cells," Proc Natl Acad Sci U S A, vol. 112, pp. E3661-8, Jul 142015. 